\title{
Emission UV des décharges capillaires mercure-gaz-rare basse pression
}

\author{
G. Zissis et J.J. Damelincourt
}

Laboratoire des Décharges dans les Gaz, URA 277, 118 route de Narbonne, 31062 Toulouse cedex, France

Résumé: La production du rayonnement ultraviolet par les décharges électriques dans les mélanges mercure-gaz-rare basse pression dépend fortement de la pression partielle de la vapeur de mercure dans le plasma ainsi que de la géométrie du système. Les résultats présentés ici, obtenus par un modèle collisionel-radiatif auto-cohérent, montrent que le maximum d'efficacité de la production du rayonnement à $254 \mathrm{~nm}$ par des tubes capillaires (quelques $\mathrm{mm}$ de diamètre) est déplacé vers les plus fortes valeurs de la pression partielle de la vapeur de mercure. Dans ce cas l'efficacité peut atteindre des valeurs comprises entre $60 \%$ et $70 \%$.

\section{INTRODUCTION}

Les décharges électriques basse pression dans les mélanges mercure-gaz-rare constituent une des plus anciennes sources de rayonnement ultraviolet incohérent. En effet, le mercure, qui est l'élément actif de la décharge, émet deux raies de résonance situées dans le domaine ultraviolet du spectre $(254 \mathrm{~nm}$ et $185 \mathrm{~nm})$. Parmi les applications de ces systèmes, la première, et la plus répandue, est le tube fluorescent destiné à l'éclairage. Pour cette application, le rayonnement à $254 \mathrm{~nm}$ est converti par l'intermédiaire d'une poudre fluorescente en rayonnement visible. Notons que l'efficacité de conversion de l'énergie électrique, fournie à la décharge, en rayonnement UV à 254 nm dans la colonne positive de la décharge est excellente: dans certains cas elle peut atteindre $70 \%$. Toutefois, on peut envisager aujourd'hui d'autres applications du rayonnement UV, telles que la photochimie, la micro-lithographie, le traitement des eaux, etc... Les décharges mercure-gaz-rare constituent des candidats potentiels comme sources UV à condition d'accrô̂tre leur densité d'émission (à $254 \mathrm{~nm}$ mais aussi à $185 \mathrm{~nm}$ ) et de diminuer leur volume tout en gardant leur efficacité.

\section{RESULTATS ET DISCUSSION}

Un problème qui se pose souvent avec ces systèmes est la variation rapide du flux UV en fonction de la pression partielle du mercure dans le plasma. Etant donné que la pression du mercure est celle de la vapeur saturante, sa variation est liée à la température du point le plus froid du tube où le mercure se condense. En outre, un autre élément déterminant est la géométrie du système (forme et dimensions du tube). Nous avons étudié ici la 
possibilité d'utiliser des décharges capillaires afin d'améliorer la production du rayonnement UV dans le domaine des pressions partielles élevées. Pour cette étude nous avons développé un code numérique auto-cohérent basé sur un modèle collisionelradiatif stationnaire. La particularité de ce code est son large domaine de validité dû à la prise en compte de plusieurs phénomènes, tels que les collisions inélastiques entre particules neutres ou l'influence du gaz rare sur l'emprisonnement du rayonnement de résonance, souvent négligés dans ce type d'études. Les résultats de la simulation ont été confirmés par l'expérience [1]. La figure 1 montre que la valeur absolue de la production du rayonnement à $254 \mathrm{~nm}$ par la décharge dépend fortement non seulement du diamètre du tube mais aussi de la pression partielle du gaz tampon (ici l'argon).

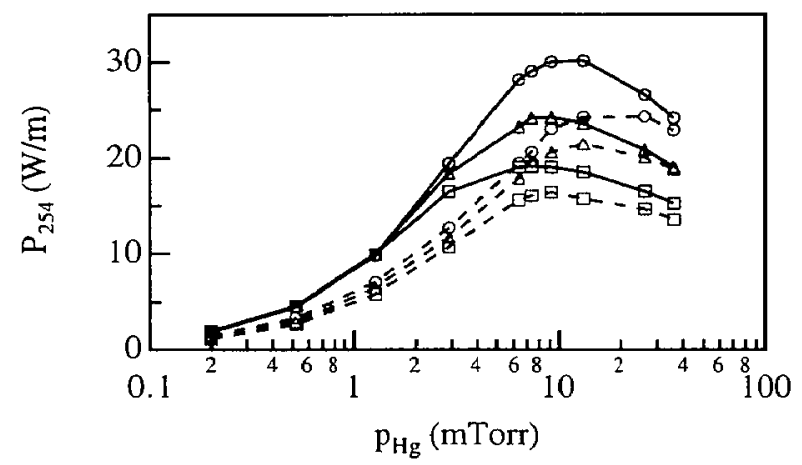

Figure 1: Production du rayonnement à $254 \mathrm{~nm}$ en fonction de la pression partielle du mercure pour deux pressions partielles d'argon ( $-: 3$ Torr, $-: 15$ Torr $)$ et trois diamètres $(\square: 36 \mathrm{~mm}, \Delta: 24 \mathrm{~mm}, O: 14 \mathrm{~mm})$.

Dans le cas précis des décharges capillaires, la figure 2 montre un net décalage, par rapport à une décharge classique, du maximum d'efficacité pour la production de la raie $254 \mathrm{~nm}$ vers les fortes pressions partielles de la vapeur saturante de mercure. Notons aussi que l'efficacité maximale des décharges capillaires reste proche de celle des décharges classiques.

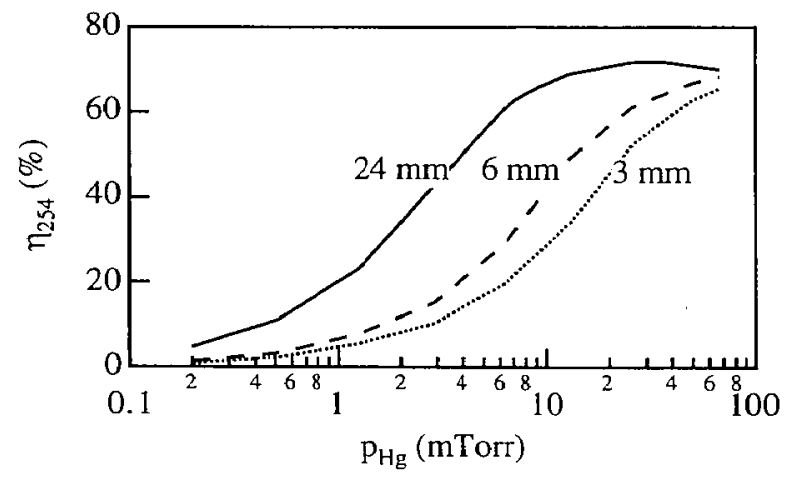

Figure 2: Efficacité lumineuse à $254 \mathrm{~nm}$ en fonction de la pression partielle du mercure pour une décharge classique $\left(\varnothing 24, I_{\text {arc }}=400 \mathrm{~mA}\right.$ ) et des capillaires ( $\varnothing 6$ et $\varnothing 3, I_{\text {arc }}=20$ $\mathrm{mA}$ ).

\section{References}

[1] Zissis G., Bénétruy P. and Bernat I., Phys. Rev. A45 (1992), 1135-1148. 